\title{
Semi-Renewable Latex via Step-Photopolymerisation of Dithiol and Dienic Terpenes
}

Bassam Tarablsia $\mathrm{PhD}$, Florent Jasinski ${ }^{\mathrm{a}} \mathrm{PhD}$, Emeline Lobry ${ }^{\mathrm{a}} \mathrm{PhD}$, Abraham Chemtob $^{\mathrm{a}^{*}} \mathrm{PhD}$, Didier Le Nouen ${ }^{\mathrm{b}} \mathrm{MS}$, Adrien Criquic PhD

a Laboratory of Photochemistry and Macromolecular Engineering, ENSCMu, University of HauteAlsace, 3 bis rue Alfred Werner 68093 Mulhouse Cedex, France

b Laboratoire de Chimie Organique et Bioorganique, ENSCMu, University of Haute-Alsace, 3 bis rue Alfred Werner, 68093 Mulhouse Cedex, France

c Mäder Research - MÄDER GROUP, 130 rue de la Mer Rouge, 68200 Mulhouse, France

Date the text was written: September 22015

Number of words (text and tables): 3226

Number of figures: 8 


\begin{abstract}
Abundance, low cost and ene functionality are the key assets of terpenes in view of a large-scale use as renewable monomers in radical polymerisation. However, their sterically hindered ene structure has been mostly ineffective in a chain-growth mechanism, leading to sluggish activity. To address this challenge, the alternative of a step-growth diene-dithiol linear photopolymerisation is investigated here. Four nonmodified dienic terpenes - geranyl acetate, linalool, limonene and geraniol - are reacted with ethylene glycol dithiol in various conditions. In bulk and solution, limited conversions prevent the formation of high-molecular-weight polymers. By contrast, a miniemulsion containing the two difunctional comonomer yields higher conversions (> $98 \%$ ) in less than 10 min of UV irradiation, making the formation of polythioether chains possible. Our assumption is that a thiol-ene polymerisation confined in nanodroplets may drive high conversion thanks to a radical compartmentalization effect. The resultant aqueous polymer dispersions show an average diameter ranging from 100 to $140 \mathrm{~nm}$, low glass transition temperature $\left(<-30{ }^{\circ} \mathrm{C}\right)$, but only moderate molecular weights between 4 and $5 \mathrm{kDa}$ for the fraction soluble in THF. These promising results pave the way for a novel semi-synthetic and ecoefficient approach to polymer latexes based on natural terpene monomers.
\end{abstract}

\title{
Keywords
}

Polymers; Macromolecule; Coatings

\section{List of notation}

is the irradiance of the UV source

$T_{g} \quad$ is the glass transition temperature

$T_{m} \quad$ is the melting temperature

$R T$-FTIR is real-time Fourier transform infrared spectroscopy

$D_{d} \quad$ is the droplet average size

$D_{p} \quad$ is the particle average size

DLS is the dynamic light scattering technique to determine droplet/particle size

SEC is size exclusion chromatography

$\bar{M}_{n} \quad$ is the number average molecular weight

$\bar{M}_{w} \quad$ is the weight average molecular weight

PDI is the polydispersity index determined by SEC

$P I \quad$ is a photoinitiator

NMR is nuclear magnetic resonance

THF is tetrahydrofuran 


\section{Introduction}

In synthetic polymerisation, most monomer precursors derive directly or indirectly from fossil fuel feedstock, mainly ethylene, acetylene and benzene. ${ }^{1}$ Finding sustainable alternatives based on renewable biomass resources has become a major societal and technological issue in effort to reduce our dependence on coal, oil and natural gas. Among the natural molecular biomass available, terpenes have been recently brought to the forefront as the possible next-generation reserve of organic monomers. ${ }^{2}$ Structured around a carbon skeleton of isoprene units (2-methyl1,4-butadiene), terpenes bear an enormous structural diversity. Ranked as one of the largest families of natural compounds, they are found in the essential oil of many plants, trees and fruits. The major source is turpentine $\left(35 \times 10^{4} \text { tons produced annually }\right)^{3}$ obtained from the distillation of live trees' resin, mainly conifers. Biodegradability, low toxicity, and bioactivity have already made terpenes suitable for manifold applications, e.g. as pharmaceuticals, insecticides, solvents, fragrances, flavours and chiral intermediates. ${ }^{4}$ By contrast, there has been less emphasis on their potential as monomer in polymerisation.

However, terpenes have in theory the required features to become a renewable natural feedstock for large-scale polymer synthesis: abundance, low cost and functionality. Despite this overall positive climate, there is currently no direct polymerisation procedure combining efficiency and broad choice of terpene monomers. The majority of the existing studies have focused on pinene (Figure 1), the most abundant monoterpene extracted from turpentine. The $\beta$-pinene enantiomer, which has a single 1,1-disubstituted exocyclic double bond with electron donating substituents, is indeed one of the few terpenes providing the requisite carbocation stability for cationic polymerisation. Cationic polymerizability of $\alpha$-phallandrene was also described. ${ }^{5}$ By contrast, all monoterpenes containing generally di- or trisubstituted insaturations (see examples in Figure 1) failed to homopolymerise by radical means because of steric effects. Nevertheless, they were able to enter into radical copolymerisation reactions with a variety of mono- or 1,1-disubstituted monomers like styrene, ${ }^{6}$ methyl methacrylate, ${ }^{7}$ acrylonitrile, ${ }^{8}$ or maleimide ${ }^{9}$ but at very sluggish reaction rates. The last strategy relied on chemical modification of terpenes to introduce more readily polymerisable groups. The most famous was the epoxidation of limonene yielding limonene (di)oxide widely reported for cationic photopolymerisation. ${ }^{10}$ Using diolefinic terpenes such as limonene as substrate, thiol-ene coupling reactions served to synthesize new homo- or hetero-bifunctional monomers bearing thiol, ${ }^{11-13}$ alcohol, ${ }^{2}$ ester, ${ }^{2}$ amine ${ }^{14,}{ }^{15}$ or alkene. ${ }^{16}$ Using these modified terpenes, a number of step-growth polymerisations were conducted, over the past four years essentially, resulting in linear or network polymers based on polyester, ${ }^{2}$ polyamide or polyurethane ${ }^{14}$ and polythioether. ${ }^{13}$

Surprisingly, the direct free-radical polyaddition of polythiols to dienic terpenes was hardly ever described, ${ }^{17}$ although this route offers several advantages. A step-growth thiol-ene polymerisation of $\mathrm{AA}$ and $\mathrm{BB}$ difunctional monomers can take advantage of a wealth of naturally 
occurring dienic terpenes (Figure 1), and create a single-step approach to "semi-synthetic" linear polythioether. The main challenge is to achieve high-yield reactions necessary to the formation of high-molecular-weight linear polymers. As detailed in Figure 2, this (photoinduced) reaction proceeds via an addition-transfer mechanism. ${ }^{18}$ The bottleneck with unmodified terpenes is that internal olefins are approximately 10 times less reactive than terminal ones toward the addition ( $\left.\mathrm{k}_{\text {add }}\right)$ of the photogenerated alkylthiyl radicals (eq 2), whereas the fragmentation $\left(\mathrm{k}_{\mathrm{frag}}\right)$ of the corresponding $\beta$-sulfanyl alkyl radical is accelerated by 2 orders of magnitude. ${ }^{19}$

To address reactivity limitation, our chosen strategy involves changing the conventional homogeneous processing in bulk or solution. Although dispersion or emulsion methods have been used rarely to make thiol-ene polymerizations, these heterogeneous techniques may displace the reaction in the direction of high-molecular-weight products. ${ }^{20,}{ }^{21}$ The main driving force is the so-called radical compartmentalization effect with a polymerisation confined within a high number of particles. ${ }^{22}$ Under these conditions, there is ideally less than one radical per particle, with the net effect of minimizing radical recombination/termination (eqs 4-6, Figure 2).

In this study, we chose to conduct thiol-ene photopolymerisation with aqueous miniemulsions containing an exact stoichiometric balance of dithiol monomer and dienic terpene. As shown in Figure 3, the reaction of four different terpenes - geraniol acetate 1, linalool 2, limonene 3, and geraniol 4 - with ethylene glycol dithiol (EGDT) has been investigated, and compared to solution and mass polymerisation. Small and colloidally stable monomer droplets with an average diameter of ca. $100 \mathrm{~nm}$ have been prepared in order to promote both compartmentalization effect and improved UV light penetration. Miniemulsions are defined as submicrometric droplets. They typically span a diameter of $50-500 \mathrm{~nm}$ and exhibit a colloidal stability of some hours up to several months. ${ }^{23}$ Due to their large surface area compared to macroemulsion, their nucleation preferentially proceeds by droplet nucleation. The radical step-growth polymerisation of terpenes in miniemulsion or in other heterogeneous conditions is unprecedented, while its chain-growth analogue has been scarcely reported. ${ }^{24,25}$ We believe that enhanced reactivity and higher molecular weight can emerge from a miniemulsion thiol-ene photopolymerization. In addition, such process fits well with current efforts to find mild, "green" processing routes to materials. The combined use of solvent-free formulation, energy-saving UV equipment and room-temperature process indeed provides avenue for a more sustainable growth.

\section{Experimental section}

\subsection{Materials}

All miniemulsions were prepared with distilled water $(\approx 6 \mathrm{M} \Omega . \mathrm{cm})$. Technical grade terpenes: geranyl acetate (97\%) (1), geraniol (97\%) (2), linalool (97\%) (3) and limonene (97\%) (4) were supplied by Alfa Aesar and used without further purification. Ethylene glycol dithiol (EGDT) (> 95 $\%)$ was supplied by Sigma-Aldrich and used without further purification. The radical photoinitiator 
1-[4-(2-Hydroxyethoxy)-phenyl]-2-hydroxy-2-methyl-1-propane-1-one (I2959) was provided by BASF Specialty Chemicals. Sodium dodecyl sulfate (SDS) (Sigma-Aldrich) is the surfactant used in this study. Hexadecane (Sigma-Aldrich) was added to the miniemulsion formulation as hydrophobe. The various solvents, tetrahydrofuran, toluene, dichloromethane and acetonitrile were provided by Sigma-Aldrich.

\subsection{Preparation and photopolymerisation of terpene-dithiol monomer miniemulsions}

\subsubsection{Monomer miniemulsion preparation}

Four different unmodified terpenes (1-4) have been investigated as regards direct thiol-ene photopolymerisation with a nonbiosourced dithiol monomer (EGDT) to form a semi-synthetic polythioether based on a conventional $\mathrm{AA}$ and $\mathrm{BB}$ difunctional monomer step-growth polymerisation. In a typical synthesis, an organic phase was first prepared by mixing $0.1 \mathrm{~g}$ of hexadecane ( $4 \mathrm{wt} . \%$ with respect to the Terpene-EGDT mixture phase) to $2 \mathrm{~g}$ of the monomer mixture (Terpene/EGDT, 1/1 mol). The aqueous phase was prepared separately by dissolving $0.075 \mathrm{~g}$ of SDS (3 wt.\% with respect to the Terpene-EGDT mixture phase) and $0.075 \mathrm{~g}$ of I2959 (3 wt.\% with respect to the Terpene-EGDT mixture phase) in $20 \mathrm{~mL}$ of distilled water. Both phases were roughly mixed during 10 min using a magnetic stirrer at $700 \mathrm{rpm}$. The coarse emulsion was then homogenized during $5 \mathrm{~min}$ in a Branson Sonifier $450(450 \mathrm{~W} / \mathrm{L})$ while maintaining the agitation.

\subsubsection{Monomer mixture for bulk polymerisation.}

$0.015 \mathrm{~g}$ of photoinitiator (I2959) (3 wt. \% with respect to the Terpene-EGDT mixture phase) was added to $(0.5 \mathrm{~g})$ of the thiol-ene mixture (Terpene/EGDT, $1 / 1 \mathrm{~mol})$. This latter was mixed during 10 min using a magnetic stirrer at 700 rpm before its deposition as thin films.

\subsubsection{Monomer mixture for solution polymerisation}

$0.2 \mathrm{~g}$ of the mixture of monomers (Terpene/EGDT, $1 / 1 \mathrm{molar}$ ) was dissolved in $0.8 \mathrm{~g}$ of organic solvent (THF, toluene, dichloromethane, acetonitrile). Then $0.006 \mathrm{~g}$ of photoinitiator (I2959) (3 wt. $\%$ with respect to the Terpene-EGDT mixture phase) was added and the mixture was mixed during $10 \mathrm{~min}$ using a magnetic stirrer at $700 \mathrm{rpm}$.

\subsection{Photopolymerisation}

\subsubsection{Miniemulsion and solution photopolymerisations}

Solution and miniemulsion photopolymerisations were performed at ambient temperature in a spectroscopic quartz cell (thickness: $1 \mathrm{~mm}$, volume: $0.34 \mathrm{~mL}$, no stirring) acting as a photochemical microreactor. Illumination was provided during $10 \mathrm{~min}$ by the polychromatic light of a medium pressure mercury-xenon arc lamp (Hamamatsu L8252, equipped with a reflector at $365 \mathrm{~nm})(\lambda$ : 250- $600 \mathrm{~nm}$, irradiance $=I=685 \mathrm{~mW} \mathrm{~cm}^{-2}$ ) focalized vertically on the sample through a flexible 
light guide placed at a distance of $4.2 \mathrm{~cm}$ from the cuvette. In solution, the photopolymerisation kinetics were monitored in situ by real-time Fourier transform infrared spectroscopy (RT-FTIR, IFS 66S from Bruker Optics) by monitoring the decay of the IR absorption bands of terpenes at different wavenumbers (depending on the reacted terpene) under simultaneous exposure to UV light. However, a similar characterization technique was not found possible in miniemulsion because of water interference. Conversions were also determined by NMR.

\subsubsection{Bulk photopolymerisation}

All the irradiation experiments were performed at ambient temperature in the presence of air. A liquid thiol-ene monomer film (10 $\mu \mathrm{m}$ thick) was applied onto a $\mathrm{BaF}_{2}$ crystal by means of a calibrated wire-wound applicator. Subsequently the films were exposed to the same UV light source as described above, and kinetics were measured by RT-FTIR as mentioned above.

\subsection{Characterization}

Monomer droplet $\left(D_{d}\right)$ size (z-average diameters) and polymer particle size $\left(D_{p}\right)$ after polymerisation were determined by Dynamic Light Scattering $(D L S)$ using a Zetasizer Nano ZS (Malvern instruments). For the sample preparation, the miniemulsion (before or after polymerisation) was diluted in distilled water, and measurements were conducted within 5 min of its preparation. The stability of the oil in water miniemulsion was monitored by measuring the transmittance and backscattering of a pulsed near infrared light $(\lambda=880 \mathrm{~nm})$ by Turbiscan Lab Expert (Formulaction). The transmittance detector received the light which passed through the dispersion at an angle of $180^{\circ}$ with respect to the source, while the backscattering detector received the light scattered backward by the dispersion at an angle of $45^{\circ}$. The thiol-ene monomer miniemulsion was placed into a cylindrical glass tube and submitted to stability analysis. The two detectors scanned the entire height of the various samples of miniemulsions at $32{ }^{\circ} \mathrm{C}$ for $4 \mathrm{~h}$. Glass transition temperature $\left(T_{g}\right)$ of the different obtained polythioethers were determined by differential scanning calorimetry (DSC). The DSC characterizations (DSC Q200, TA Instruments) were performed under nitrogen atmosphere $(50 \mathrm{~mL} / \mathrm{min})$ with ca. $8 \mathrm{mg}$ of dried latex placed in an aluminium hermetic pan and heated at $10{ }^{\circ} \mathrm{C} \mathrm{min}^{-1}$. Number-average molecular weights $\left(\overline{M_{n}}\right)$, weight-average molecular weights $\left(\overline{M_{w}}\right)$ and molecular weight distributions $\left(\overline{M_{w}} / \overline{M_{n}}\right)$ were determined by Size Exclusion Chromatography (SEC) coupled on line to a refractive index (RI) (Shimatzu RID-10A) and ultra-violet (SPD-10Avp) detectors, and equipped with a series of four PLgel $5 \mu \mathrm{m}$ Mixed-B columns (separation domain: 100 - $60000 \mathrm{~g} \mathrm{~mol}^{-1}$ ). The mobile phase was THF with a flow rate of $1 \mathrm{~mL} \mathrm{~min}{ }^{-1}$ using a Shimadzu LC20AD pump. Toluene was used as internal reference. The SEC results were obtained using a fixed $\mathrm{dn} / \mathrm{dc}$ value and a molecular weight calibration based on 10 narrow molecular weight standards of linear polystyrene from Polymer lab. The $\overline{M_{n}}$ and $\overline{M_{w}} / \overline{M_{n}}$ values displayed in this manuscript were measured with the RI detector. ${ }^{1} \mathrm{H}$ NMR (400 MHz) and ${ }^{13} \mathrm{C}$ NMR (100 MHz) spectra were recorded on a Bruker Avance 400 
spectrometer equipped with Ultrashield magnet, and chemical shifts were referenced to the residual proton signal of the solvents $\mathrm{CHCl}_{3}$ at $7.26 \mathrm{ppm}$.

\section{Results and discussion}

All four dienic terpenes (1-4) are unable to homopolymerize upon UV light either in solution or in bulk in the presence of a hydroxyl acetophenone radical photoinitiator (I2959) (see Fig. S1 of the supplementary data). In contrast, the addition of a $\omega, \omega^{\prime}$-dithiol monomer (EGDT) to get an exact thiol-ene stoichiometric balance drives a dramatic increase in conversion and polymerisation rate as illustrated in Figure 4 in the case of geranyl acetate 1. These RT-FTIR kinetic plots performed in bulk and in solutions using a range of solvents show the added-value of a radical step-growth process. Proceeding in bulk or in low UV absorbing solvents such as $\mathrm{CH}_{2} \mathrm{Cl}_{2}$ provides the most favourable reactions conditions, leading respectively to $87 \%$ and $58 \%$ conversion in ene functions after 10 min exposure. However, these levels of conversion remain insufficient to produce polymer products in a step-reaction, but only dimer to pentamer structures. Similarly, limiting ene

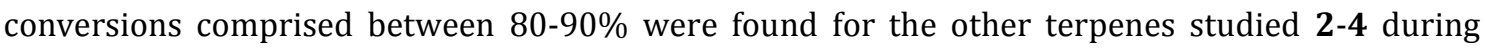
their bulk photopolymerization (see Fig S2. in supplementary data). From the practical standpoint, it should be realized that the successful synthesis of high-molecular-weight linear polymers using any step-growth polymerisation reaction can be achieved only at very high conversions (> 98-99 $\%)$.

Alternatively, the initial 1-EGDT organic mixture can serve to prepare a miniemulsion stabilized by hexadecane (hydrophobe to overcome Ostwald ripening) and SDS (surfactant to promote emulsification and droplet stabilization). This miniemulsion contains also the same photoinitiator (I2959) used in the aforementioned bulk or solutions formulations, but this time dissolved in the aqueous phase (see the experimental section for details on preparation method). An average droplet diameter of $120 \mathrm{~nm}$, remaining stable during several hours, was evaluated by DLS. In addition, the colloidal stability of this miniemulsion was conclusively assessed by backscattering measurements, showing minimal changes of reflectance within 4 hours of preparation as shown in Figure 5.

The thiol-ene monomer droplets were eventually irradiated at ambient temperature in a $1 \mathrm{~mm}$ thick reactor during $10 \mathrm{~min}$ with affecting the colloidal stability. Evidence of polymerisation was investigated by analysing the resulting sticky material (noted P1) obtained after water evaporation using NMR and SEC. In contrast to the bulk films, FTIR was of limited utility because of high water concentration causing signal saturation. The ${ }^{1} \mathrm{H}-\mathrm{NMR}$ spectra of the two monomers (EGDT and 1) and the polymer are shown in Figure 6. First, we note a significant conversion of the two reactive groups in P1: the two ethylenic proton signals $\mathrm{H} 6$ and $\mathrm{H} 2$ (1, 5.00 and $5.20 \mathrm{ppm}$ ) have almost disappeared, while the residual thiol proton resonances H13 (EGDT, $1.65 \mathrm{ppm}$ ) are difficult to detect in a region where other protons arise. Based on the distinctive ethylenic protons, a 
conversion of $99 \%$ was determined. In addition, the formation of thioether bonds $\left(-\mathrm{CH}_{2}-\mathrm{S}-\right)$ is noticeable in the $\mathbf{P 1}$ spectrum displaying a broad signal assigned to these particular aliphatic protons (H2', H6' and H12' between 2.5 and $2.8 \mathrm{ppm}$ ). Furthermore, a SEC analysis of the dried latex (Figure 7) was carried out in THF. However, a weight fraction of approximately $30 \%$, turned out to be insoluble in THF, and could not be analysed by SEC. The soluble fraction, presumably assigned to the lowest molecular weight portion, revealed moderate molecular weights $\left(\overline{M_{n}}\right)$ comprised between 4 and $6 \mathrm{kDa}$ (see Table 1) with the residual presence of low-molecular-weight species ranging from dimers $\left(\overline{M_{n}}=0.31 \mathrm{kDa}\right)$ to pentamers $\left(\overline{M_{n}}=1.45 \mathrm{kDa}\right)$. Such oligomers may account for the residual presence of ethylenic protons in the NMR spectrum of P1. In a step-growth polymerization, it is established that any deviation from an ideal 1:1 molar ratio in reactive groups or the formation of cyclic compounds may decrease molecular weight. We believe that the main obstacle to high-molecular-weight polymers is the difficulty to have a stoichiometric balance in thiol and ene groups. The first factor is direct and related to the limited purity of the monomers (EGDT: $>95 \%$ and terpene > $97 \%$ ). The second source of stoichiometric unbalance is that each individual droplet is likely to deviate compositionally from the stoichiometric because of the more pronounced water-solubility of EGDT (15 g/L) compared to the oil-soluble terpenes. However, the fact that high MW polymer can be achieved in miniemulsion evidences that the thiol reactant may diffuse from the aqueous phase to the particle and undergo polymerization.

Despite the relatively low molecular weight, the main achievement is the ability to obtain linear polymer products from the EGDT-1 thiol-ene polymerization. Additionally, there are significant improvements as regards reactivity and molecular weight compared to bulk or solution process, thus emphasizing the relevance of a thiol-ene reaction carried out in emulsion. The difficulty with internal ene monomers like terpene is the importance of fragmentation over addition that inhibits and slowdowns the propagation reaction, thereby increasing the risk of terminations. In thiol-ene chemistry, there are indeed a number of possible termination pathways through recombination of carbon and thiyl radicals (eqs 4-6, Figure 2). Compared to homogenous process, segregation of a propagating radical in a polymer particle isolates it from other propagating radicals and allows rate and degree of polymerisation to be simultaneously increased, provided a high number of polymer particles are generated. This important reaction characteristic has been widely reported in chain radical polymerisation, and seems to be also applicable to a step radical process.

Regarding colloidal characterization of the latex derived from P1, Figure 8 shows a comparison between the droplet and particle size distribution, respectively before and after irradiation. Obviously, there is a significant match between both size profiles, which may be the sign that a high proportion of the initial monomer droplets has been nucleated to give birth to a particle. The synthesized polysulfide P1 was also characterized thermally by means of DSC. A low glass transition temperature $\mathrm{T}_{\mathrm{g}}$ value of at $-47^{\circ} \mathrm{C}$, which is consistent with the flexible and long C-S bonds arising from a thiol-alkene addition reaction. Preliminary results gathered in Table 1 show 
that this concept of miniemulsion photopolymerisation could be extended to other dienic terpenes 2-4 leading to high conversions in all instances. Relatively lower $\overline{M_{n}}$ values were obtained with 2 (see SEC trace in Fig S3.). However, the dienes $\mathbf{3}$ and $\mathbf{4}$ yielded polythioether fully insoluble in THF, preventing their analysis by SEC. Although poorly soluble in $\mathrm{CDCl}_{3}$, their analysis was found possible through NMR, which revealed conversions of $98 \%$ (P3) and $95 \%$ (P4) (see spectra in Fig S4-S6). It has been hypothesized that polymer insolubility may originate from the presence of crystalline domains. The semi-crystallinity of linear poly(thioether) has been reported in the literature, in particular as regards with poly(alkylene sulphide), ${ }^{26}$ and attributed to chain linearity (no branching) and flexibility, both conducive to chain packing. Unfortunately, the DSC is of limited utility because it does not show polymer melting peaks. It is reasonable to postulate that the melting point is too high to be observed before the thermal decomposition which occurs around $200^{\circ} \mathrm{C}$. Obviously, the use of wide-angle X-ray diffraction (WAXD) should shed further light in the future on this important issue.

\section{Conclusion}

When involving terpenes in a thiol-ene photopolymerisation, the reaction rate is significantly affected by the internal ene structure. By contrast, we found that that semi -renewable latexes can be successfully synthesized using four non-modified dienic terpenes through miniemulsion photopolymerisation in presence of EGDT. The polymerisation in miniemulsion demonstrated two major advantages over the bulk and solution polymerisation: high monomer (>95\%) conversions and the generation of polythioether products. We postulate that the compartmentalization effect unique to emulsion polymerization process is responsible for the formation polymer products. The molecular weights remained relatively low, although the validity of this result was mitigated by poor or no solubility. This result points out the necessity of finding new means to drive conversion towards higher values, by adjusting ene/thiol proportion, droplet size, thiol structure. Alternatively, these low-molecular weight polythioether, with terminal ethylenic or thiol functionalities, could be used as prepolymer materials for further polymerization reactions.

\section{Acknowledgements}

We would like to thank Mélanie Legros (Institut Charles Sadron, University of Strasbourg) for helpful discussions on SEC results. Financial support by French National Research Agency (ANR, Programme Chimie Durable-Industries-Innovation under contract number ANR-2012-CDII-006-02) is gratefully acknowledged.

\section{References}

1. Wilbon, P. A.; Chu, F.; Tang, C. Progress in Renewable Polymers from Natural Terpenes, Terpenoids, and Rosin, Macromol Rapid Commun. 2013, 34, 8-37.

2. $\quad$ Firdaus, M.; de Espinosa, L. M.; Meier, M. A. R. Terpene-Based Renewable Monomers and Polymers via Thiol-Ene Additions, Macromolecules. 2011, 44, 7253-7262. 
3. Morris, M. The Global Supply Position for Turpentine, Proccedings of Conference The Global Supply Position for Turpentine, Florida, USA, 2000, 17-31.

4. $\quad$ Eggersdorfer, M. Terpenes. In Ullmann's Fine Chemicals, VCH-Wiley, 2014, 1185-1202.

5. Satoh, K.; Sugiyama, H.; Kamigaito, M. Biomass-derived heat-resistant alicyclic hydrocarbon polymers: poly(terpenes) and their hydrogenated derivatives, Green Chem. 2006, 8, 878-882.

6. Barros, M. T.; Petrova, K. T.; Ramos, A. M. Potentially Biodegradable Polymers Based on $\alpha-$ or $\beta$-Pinene and Sugar Derivatives or Styrene, Obtained under Normal Conditions and on Microwave Irradiation, Eur J Org Chem. 2007, 8, 1357-1363.

7. Ramos, A. M.; Lobo, L. S. Polymers from pine gum components: Radical and coordination homo and copolymerization of pinenes, Macromol Symp. 1998, 128, 43-50.

8. Li, A.-L.; Wang, Y.; Liang, H.; Lu, J. Controlled radical copolymerization of $\beta$-pinene and acrylonitrile, J Polym Sci, Part A: Polym Chem. 2006, 44, 2376-2387.

9. Satoh, K.; Matsuda, M.; Nagai, K.; Kamigaito, M. AAB-Sequence Living Radical Chain Copolymerization of Naturally Occurring Limonene with Maleimide: An End-to-End SequenceRegulated Copolymer, J Am Chem Soc. 2010, 132, 10003-10005.

10. Park, H. J.; Ryu, C. Y.; Crivello, J. V. Photoinitiated cationic polymerization of limonene 1,2oxide and a-pinene oxide, J Polym Sci, Part A: Polym Chem. 2013, 51, 109-117.

11. Marvel, C. S.; Olson, L. E. Polyalkylene sulfides. XIII. Polymers from 4-vinyl-1-cyclohexene and d-limonene, J Polym Sci. 1957, 26, 23-28.

12. Yan, J.; Ariyasivam, S.; Weerasinghe, D.; He, J.; Chisholm, B.; Chen, Z.; Webster, D. Thiourethane thermoset coatings from bio-based thiols, Polym Int. 2012, 61, 602-608.

13. Firdaus, M.; Meier, M. A. R.; Biermann, U.; Metzger, J. O. Renewable co-polymers derived from castor oil and limonene, Eur J Lipid Sci Techno. 2014, 116, 31-36.

14. Firdaus, M.; Meier, M. A. R. Renewable polyamides and polyurethanes derived from limonene, Green Chem. 2013, 15, 370-380.

15. Alves, M. H.; Sfeir, H.; Tranchant, J. F.; Gombart, E.; Sagorin, G.; Caillol, S.; Billon, L.; Save, M. Terpene and Dextran Renewable Resources for the Synthesis of Amphiphilic Biopolymers, Biomacromolecules. 2014, 15, 242-251.

16. Claudino, M.; Mathevet, J.-M.; Jonsson, M.; Johansson, M. Bringing D-limonene to the scene of bio-based thermoset coatings via free-radical thiol-ene chemistry: macromonomer synthesis, UVcuring and thermo-mechanical characterization, Polym Chem. 2014, 5, 3245-3260.

17. Hearon, K.; Nash, L. D.; Rodriguez, J. N.; Lonnecker, A. T.; Raymond, J. E.; Wilson, T. S.; Wooley, K. L.; Maitland, D. J. A High-Performance Recycling Solution for Polystyrene Achieved by the Synthesis of Renewable Poly( thioether) Networks Derived from d-Limonene, Adv Mater. 2014, 26, 1552-1558.

18. Claudino, M.; Jonsson, M.; Johansson, M. Thiol-ene coupling kinetics of D-limonene: a versatile 'non-click' free-radical reaction involving a natural terpene, $R S C A d v . \mathbf{2 0 1 3}, 3,11021$ 11034.

19. Denes, F.; Pichowicz, M.; Povie, G.; Renaud, P. Thiyl Radicals in Organic Synthesis, Chem Rev. 2014, 114, 2587-2693.

20. Jasinski, F.; Lobry, E.; Tarablsi, B.; Chemtob, A.; Croutxé-Barghorn, C.; Le Nouen, D.; Criqui, A. Light-Mediated Thiol-Ene Polymerization in Miniemulsion: A Fast Route to Semicrystalline Polysulfide Nanoparticles, Acs Macro Lett. 2014, 3, 958-962.

21. Lobry, E.; Jasinski, F.; Penconi, M.; Chemtob, A.; Croutxe-Barghorn, C.; Oliveros, E.; Braun, A. M.; Criqui, A. Continuous-flow synthesis of polymer nanoparticles in a microreactor via miniemulsion photopolymerization, $R S C A d v$. 2014, 4, 43756-43759.

22. Cunningham, M. F. Living/controlled radical polymerizations in dispersed phase systems, Prog Polym Sci. 2002, 27, 1039-1067.

23. Asua, J. M. Challenges for industrialization of miniemulsion polymerization, Prog Polym Sci. 2014, 39, 1797-1826.

24. Johanson, A. J.; McKennon, F. L.; Goldblatt, L. A. Emulsion polymerization of myrcene, Ind Eng Chem. 1948, 40, 500-501.

25. Marvel, C. S.; Hwa, C. C. L. Polymyrcene, J Polym Sci. 1960, 45, 25-34.

26. Sasanuma, Y.; Hayashi, Y.; Matoba, H.; Touma, I.; Ohta, H.; Sawanobori, M.; Kaito, A. Conformational Analysis of Poly(propylene sulfide), Macromolecules. 2002, 35, 8216-8226. 
Table 1.

\begin{tabular}{|c|c|c|c|c|c|c|}
\hline Terpene & $\begin{array}{c}\text { Droplet/Particle } \\
\text { diameter } \\
(\mathrm{nm})\end{array}$ & $\begin{array}{c}\text { Ene } \\
\text { Conversion } \\
(\%)^{\mathrm{a})}\end{array}$ & $\begin{array}{c}\mathrm{T}_{\mathrm{g}} \\
\left({ }^{\circ} \mathrm{C}\right)^{\mathrm{b})}\end{array}$ & $\begin{array}{c}\overline{M_{n}} \\
(\mathrm{kDa})^{\mathrm{c})}\end{array}$ & $\begin{array}{c}\overline{M_{w}} \\
(\mathrm{kDa})^{\mathrm{c})}\end{array}$ & PDI $^{\mathrm{c})}$ \\
\hline $\mathbf{1}$ & $120 / 120$ & 99 & -47 & 5.2 & 12.3 & 2.8 \\
\hline $\mathbf{2}$ & $140 / 140$ & 99 & -43 & 3.7 & 7.4 & 2.6 \\
\hline $\mathbf{3}$ & $150 / 100$ & 98 & -41 & $-\mathrm{-d})$ & - & - \\
\hline $\mathbf{4}$ & $100 / 140$ & $95^{\mathrm{e})}$ & -34 & $-\mathrm{-d})$ & - & - \\
\hline
\end{tabular}

a) Conversion determined by ${ }^{1} \mathrm{H}$ NMR in $\mathrm{CDCl}_{3}$

b) DSC data

c) SEC data

d) P3 and $\mathbf{P 4}$ were found to be insoluble in THF

e) Obtained after a longer time of irradiation (20 min) 